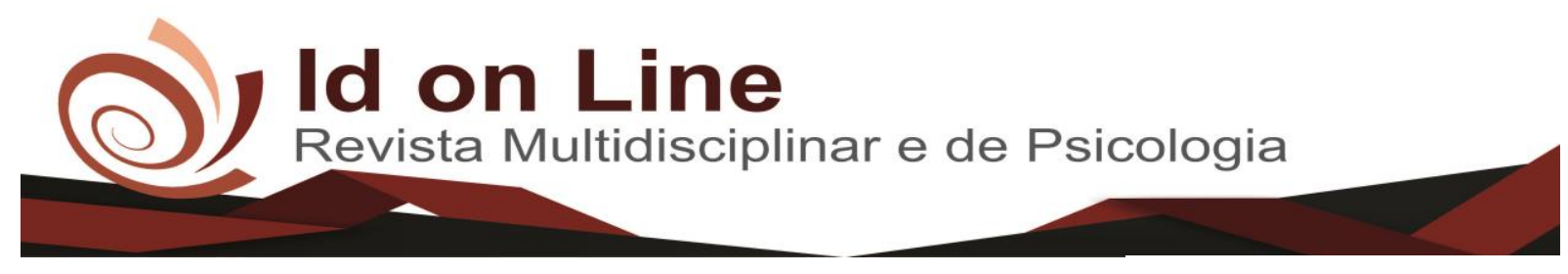

Artigo de Revisão

\title{
Cuidadores de Idosos e o Déficit no Autocuidado
}

\author{
Raquel Gouveia Ramos ${ }^{1}$; Claudia Daniele Barros Leite-Salgueiro ${ }^{2}$; Juliane da Silva Pereira ${ }^{3}$; \\ Luciclaudio da Silva Barbosa ${ }^{4}$; Luzineide Lobato
}

Resumo: Os idosos são mais vulneráveis para o aparecimento de patologias e junto com o envelhecimento, muitas alterações fisiológicas acontecem, com isto, muitos acabam ficando dependentes e necessitando de cuidados e auxílio de outra pessoa para a realização de suas atividades básicas. O principal objetivo do presente estudo, foi identificar como os estudos científicos têm abordado a saúde mental do cuidador de idosos e a relação disso com a sobrecarga de atividades. Trata-se de uma revisão integrativa da literatura. Para a realização da pesquisa, foram utilizados meios eletrônicos como as seguintes bases de dados: LILACS, SciELO e BDENF, que foram acessados pela Biblioteca Virtual em Saúde (BVS). Os atores referenciados encontraram grandes índices de sobrecarga aos cuidadores de idosos, o que causa um declínio em sua qualidade de vida e principalmente na saúde mental dos mesmos.

Descritores: Cuidador; família; idosos; saúde mental.

\section{Caregivers of Elderly and Non-Self-Covered Deficit}

Abstract: The elderly are more vulnerable to the appearance of pathologies and along with aging, many physiological changes happen, with this, many end up becoming dependent and needing care and assistance of another person to perform their basic activities. The main objective of the present study was to identify how scientific studies have addressed the mental health of caregivers of the elderly and their relation to the overload of activities. This is an integrative review of the literature. To perform the research, electronic media were used as the following databases: LILACS, SciELO and BDENF, which were accessed by the Virtual Health Library (BVS). The referenced actors found high rates of overload to caregivers of the elderly, which causes a decline in their quality of life and especially in their mental health.

Keywords: Caregiver; family; seniors; mental health.

\footnotetext{
${ }^{1}$ Discente do Bacharelado em Enfermagem do Instituto Federal de Educação, Ciência e Tecnologia (IFPE), Campus Pesqueira. Contato: raquelgr8@hotmail.com .

${ }^{2}$ Doutora em Psicologia Clínica pela Universidade Católica de Pernambuco (UNICAP), Mestra em Ciências da Saúde pela Universidade de Pernambuco (UPE), Pós Graduada (Lato Sensu) em Saúde Coletiva pela mesma Universidade. Psicóloga formada pela Universidade Católica de Pernambuco (UNICAP). Docente do curso Bacharelado em Enfermagem do Instituto Federal de Educação, Ciência e Tecnologia de Pernambuco (IFPE) - Campus Pesqueira. Contato: claudia.leite@pesqueira.ifpe.edu.br.

${ }^{3}$ Enfermeira formada pelo Instituto Federal de Educação, Ciência e Tecnologia de Pernambuco (IFPE) - Campus Pesqueira. Assistente de saúde da Prefeitura Municipal de Pesqueira-Pe. Contato: julianedasilvapereira@gmail.com .

${ }^{4}$ Mestre em Recursos Naturais pela Universidade Federal de Campina Grande (UFCG). Biólogo formado pela Universidade Estadual da Paraíba (UEPB). Docente do Curso de Bacharelado em Enfermagem do Instituto Federal de Educação, Ciência e Tecnologia de Pernambuco (IFPE) - Campus Pesqueira. Coordenador dos laboratórios de morfofisiologias dos processos vitais em enfermagem do Instituto Federal de Educação, Ciência e Tecnologia de Pernambuco (IFPE)-Campus Pesqueira. Contato: luciclaudio@pesqueira.ifpe.edu.br ;

${ }^{5}$ Biológa (UFRN), Docente e Pesquisadora do Instituto Federal de Educação, Ciência e Tecnologia de Pernambuco (IFPE) - Campus Pesqueira. Mestra em Psicobiologia pela Universidade Federal do Rio Grande do Norte (UFRN). Recife (PE), Brasil. E-mail: luzineidelobato@ gmail.com
} 


\section{Introdução}

É considerado idoso, o indivíduo com idade igual ou superior a 60 anos. Com o aumento da expectativa de vida, consequentemente, cresce também o número de idosos. Junto com o envelhecimento, algumas alterações fisiológicas e biológicas surgem, acometendo os indivíduos em sua funcionalidade física e mental, em seu meio social e interpessoal (VIEIRA et al, 2012).

Este público é vulnerável ao aparecimento de patologias e agravos a saúde, com isso, aumenta-se o número de internações hospitalares. Para reduzir os custos com assistência hospitalar e institucional, as famílias optam pela permanência destes idosos na sua residência, sob seus cuidados (NOVAIS et al, 2013).

Com o aumento da população idosa, consequentemente o número de cuidadores dos mesmos tende a crescer, estes precisam compreender a vivência e as necessidades deste público. Sendo assim, torna-se necessário que os programas de saúde para o idoso também sejam voltados para o cuidador (OLIVEIRA E D'ELBOUX, 2012).

O processo de envelhecimento remete a mudanças biopsicossociais naturais pela passagem do tempo, em que ocorrem alterações morfofisiológicas, bioquímicas e psicológicas, causando diminuição da capacidade funcional, podendo variar de pessoa para pessoa (BRASIL, 2014).

As modificações mais frequentes são os problemas de visão, audição, diminuição das funções pulmonares e renais, déficit na atividade do sistema nervoso central e ossos mais frágeis. Esses efeitos devem ser entendidos pois, podem ser causas de complicações futuras (FALEIROS et al, 2015).

Quando o idoso apresenta dificuldades ou limitações no desempenho de suas atividades, é necessário que o mesmo seja acompanhado em sua rotina por um cuidador (LOUREIRO et al, 2014). Este pode ser tanto primário (principal, que tem maior responsabilidade sob os cuidados ao idoso), quanto secundário (auxilia o cuidador principal em tarefas complementares), como pode ser também, cuidador informal ou formal, sendo o primeiro não remunerado. É designado ao cuidador informal, aquele que há maior proximidade e apto para isto, não precisando ter experiência (ROCHA E PACHECO, 2013).

$\mathrm{Na}$ maioria das vezes, quem assume a responsabilidade de prestar o cuidado , é alguém da família, mas também pode ser um amigo, vizinho, desconhecido, sendo de forma remunerada 
ou não (LOUREIRO et al, 2014; VIEIRA et al, 2012). Este assume o comprometimento pelo cuidado, tornando-se uma ponte entre o idoso, a família e a equipe de saúde. Quando o cuidado é feito pelos cônjuges que envelhecem cuidando de seu parceiro, geralmente de idade mais avançada, eles estão mais susceptíveis a sobrecargas e problemas de saúde (VIEIRA et al, 2012).

O cuidador é o responsável pela execução de atividades diárias como: higiene pessoal, alimentação, passeios, idas a consultas, medicamentos, independente da gravidade da doença (CARDOZO et al, 2012).

Assumem o papel de cuidar do idoso por um longo período, até a morte na maior parte das vezes. Para isto, abrem mão de suas atividades de lazer e de autocuidado, colocando sua qualidade de vida em segundo plano para prestar maior cuidado ao idoso (OLIVEIRA E D'ELBOUX, 2012).

$\mathrm{Na}$ grande maioria dos casos, as atividades de cuidado em saúde são realizadas sem suporte ideal, assim, o cuidador também adoece devido à sobrecarga que enfrenta (CARDOZO et al, 2012). Entretanto, não é necessário que o idoso esteja doente para que tenha um cuidador. Com o envelhecimento, tarefas que antes eram consideradas banais, passam a ser difíceis de serem realizadas, dependendo assim, de outra pessoa para suprir estes deveres, ou auxiliar no seu desenvolvimento (SANTOS E KOETZ, 2017).

Sobremaneira, o cuidar informal gera estresse, devido à situação de ter que lidar com um idoso dependente, mudando totalmente a vida do cuidador (ROCHA \& PACHECO, 2013). Neste sentido, o cuidador familiar precisa se adaptar às mudanças que ocorrem na vida do idoso. As tarefas que lhes são concedidas necessitam de competência, habilidade e responsabilidade, gerando um grande encargo psicológico que acaba afetando sua saúde, podendo desenvolver uma doença mental (RODRIGUES et al, 2014).

Quando o idoso é portador de doença crônica, seu familiar cuidador apresenta mais sintomas como ansiedade, depressão e estresse, em comparação com a população em geral (FERREIRA et al, 2012).

Dados publicados pelo World Alzheimer Report, estimaram que em 2015 havia 46,8 milhões de pessoas com demência e que, em 20 anos, esse número será praticamente o dobro. Como consequência da doença, o indivíduo apresenta perdas cognitivas, déficit na capacidade física e mental, necessitando da presença de alguém que lhe preste cuidados (LIMA et al, 2017). 
Dentre as doenças crônico-degenerativas com maior prevalência em idosos, o câncer é a que se sobressai sob as demais. Devido ao diagnóstico tardio e com a doença já instalada e avançada, o que está ao alcance da maior parte da população é o tratamento quimioterápico, o mesmo acaba causando consequências indesejáveis como alterações físicas e efeitos colaterais podendo reagir de diversas formas e de difícil controle, assim, demandam cuidados domiciliares e como na maioria dos casos, é algum familiar que se torna responsável por estes cuidados (ANJOS E ZAGO, 2014).

A política de atenção ao idoso incentiva a prática do cuidado em casa, para que o resultado tenha maior eficácia, pois é um local com os próprios valores culturais e, estar próximo a família é considerada a melhor opção tendo em vista a autonomia e a dignidade do indivíduo (ANJOS E ZAGO, 2014).

Todavia, o responsável pelos cuidados voltados ao idoso é uma pessoa sobrecarregada, principalmente em casos de idosos considerados dependentes. Isso acontece devido ao elevado número e tipos de tarefas que lhe são postas, pois se sente obrigado de estar disponível o dia inteiro para auxílio na realização de todas as tarefas e incentivo quando o idoso não quiser realizá-las. Além disso, há as responsabilidades voltadas à casa e à família (VIEIRA et al, 2012; OLIVEIRA E D'ELBOUX, 2012).

Assim, muitas vezes os cuidadores familiares precisam deixar suas vidas de lado, para colocar a do outro em primeiro lugar, principalmente quando são idosos dependentes, restringindo o cuidador em relação a sua própria saúde (RODRIGUES et al, 2014; NOVAIS et al, 2013; CARDOZO et al, 2012).

Quando há apenas um indivíduo responsável pelos cuidados, há muita dificuldade, sobrecarga e cobrança nessa lida. Também, mais esforços para os cuidados que exigem força, como transferências e banhos, causando ainda mais cansaço físico e psicológico. Estas atividades diárias básicas ocupam muito tempo, cerca de cinco horas por dia, isto sem auxílio de equipamentos adequados para a realização das tarefas mais pesadas, por precisar doar tanto sua estrutura física, pode causar danos à saúde física e mental do cuidador (CARDOZO et al, 2012).

As situações na qual o cuidador familiar está inserido causam tensão no ambiente e, se o mesmo não estiver emocionalmente preparado, pode desencadear conflitos no meio familiar e resultados insatisfatórios da sua função, devido ao seu desgaste psicológico, a perda de poder 
aquisitivo e cansaço físico, podendo até chegar a haver maus-tratos com o idoso (FALEIROS et al, 2015).

Neste sentido, diante do crescente número de idosos e da necessidade de representação de prestadores de cuidados formais, os profissionais de saúde devem estar preparados para atender a demanda de pessoas dependentes, principalmente os idosos que estão cada vez mais presentes na população, este atendimento não deve só contemplar o modelo biomédico, e sim englobar no processo de aprendizado e capacitação, os cuidadores e/ou as pessoas que irão cuidar deste indivíduo em seu domicílio para que os mesmos saibam os procedimentos básicos para melhora na qualidade de vida (VIEIRA et al, 2012; OLIVEIRA E D'ELBOUX, 2012).

Principalmente na Atenção básica, deve haver uma atenção maior pelos profissionais de saúde acerca dos sinais de declínio na saúde mental que os cuidadores podem estar apresentando, a começar pela angústia. Ao melhorar a qualidade de vida de quem cuida, consequentemente melhorará a de quem é cuidado (ROCHA E PACHECO, 2013; (SANTOS E KOETZ, 2017).

Considera-se que a rede de apoio psicossocial pode ser uma forma terapêutica para levar benefícios à saúde do idoso e do seu cuidador. Esta rede preconiza os relacionamentos sociais entre os idosos e cuidadores, para dividir experiências e se sentirem amados (COSTA et al, 2013).

\section{Metodologia}

Esta é uma revisão integrativa da literatura. O método foi escolhido devido à necessidade de se obter dados embasados em evidências científicas. Por intermédio deste tipo de revisão, a pesquisa científica na área da saúde, especialmente no campo da enfermagem, ganhara uma metodologia significativa para a aplicabilidade da Prática Baseada em Evidencia (PBE). Desta forma, bem executada, se distingue das demais metodologias, por ser uma ferramenta altamente articulada e subsidiada em fontes confiáveis, de modo simples e prático (SOUZA et al, 2010).

A presente revisão integrativa da literatura fora articulada em seis etapas, a saber: 1 . Identificação do tema e seleção da hipótese ou questão de pesquisa para a elaboração da revisão integrativa; 2. Estabelecimento de critérios para inclusão e exclusão de estudos/ amostragem ou 
busca na literatura; 3. Definição das informações a serem extraídas dos estudos selecionados/ categorização dos estudos; 4. Avaliação dos estudos incluídos na revisão integrativa; 5. Interpretação dos resultados; e para finalizar, 6. Apresentação da revisão/síntese do conhecimento (SOUZA et al, 2010).

Fora eleita a seguinte pergunta norteadora: "como os estudos científicos têm abordado a saúde mental do cuidador de idosos e qual a relação com a sobrecarga de atividades? “

Para a realização da pesquisa, foram utilizados meios eletrônicos, como as seguintes bases de dados: LILACS (Literatura Latino Americana e do Caribe em Ciência da Saúde), SciELO (Scientific Electronic Library Online) e BDENF (Banco de Dados em Enfermagem), que foram acessados pela Biblioteca Virtual em Saúde (BVS). Através dos seguintes descritores de saúde (DECs): Idoso, família, cuidador e saúde mental.

Estabeleceu-se os seguintes critérios de inclusão: artigos publicados entre os anos de 2012 e 2017, no idioma português, disponíveis de forma gratuita nas bases de dados citadas e na íntegra, bem como os que possuíam os objetivos referentes ao tema. Após a leitura dos artigos foram excluídos os que não tinham conteúdo compatível com o tema.

Com relação a critérios robustos de análise de produções científicas, evoca-se o atributo nível de evidência científica, avaliado na quarta etapa da divisão da revisão integrativa da literatura. Nesta fase, são avaliados os estudos segundo suas características através da prática baseada em evidências (PBE), que é um sistema de classificação de evidências caracterizado de forma hierárquica correlacionada à abordagem metodológica adotada. Estas evidências estão divididas em:

- Nível 1: evidências resultantes da meta-análise de múltiplos estudos clínicos controlados e randomizados;

- Nível 2: evidências obtidas em estudos individuais com delineamento experimental;

- Nível 3: evidências de estudos quase-experimentais;

- Nível 4: evidências de estudos descritivos (não-experimentais) ou com abordagem qualitativa;

- Nível 5: evidências provenientes de relatos de caso ou de experiência;

- Nível 6: evidências baseadas em opiniões de especialistas (SOUZA et al, 2010).

O trajeto da pesquisa encontra-se editado na Figura 1, onde também se encontra a seguinte leitura: número total de artigos recuperados (45), número total de artigos após leitura 
minuciosa e aplicabilidade de critérios de inclusão e aproximação à pergunta norteadora (13 estudos). Também há menção às bases de dados e ao quantitativo de artigos excluídos e incluídos na pesquisa. Para a pesquisa, foram combinados os descritores cuidador, família, idosos e saúde mental, utilizando o unicamente o booleano AND.

Figura 1: Fluxograma de artigos incluídos e excluídos na pesquisa com base e GALVÃO et. al. (2015).

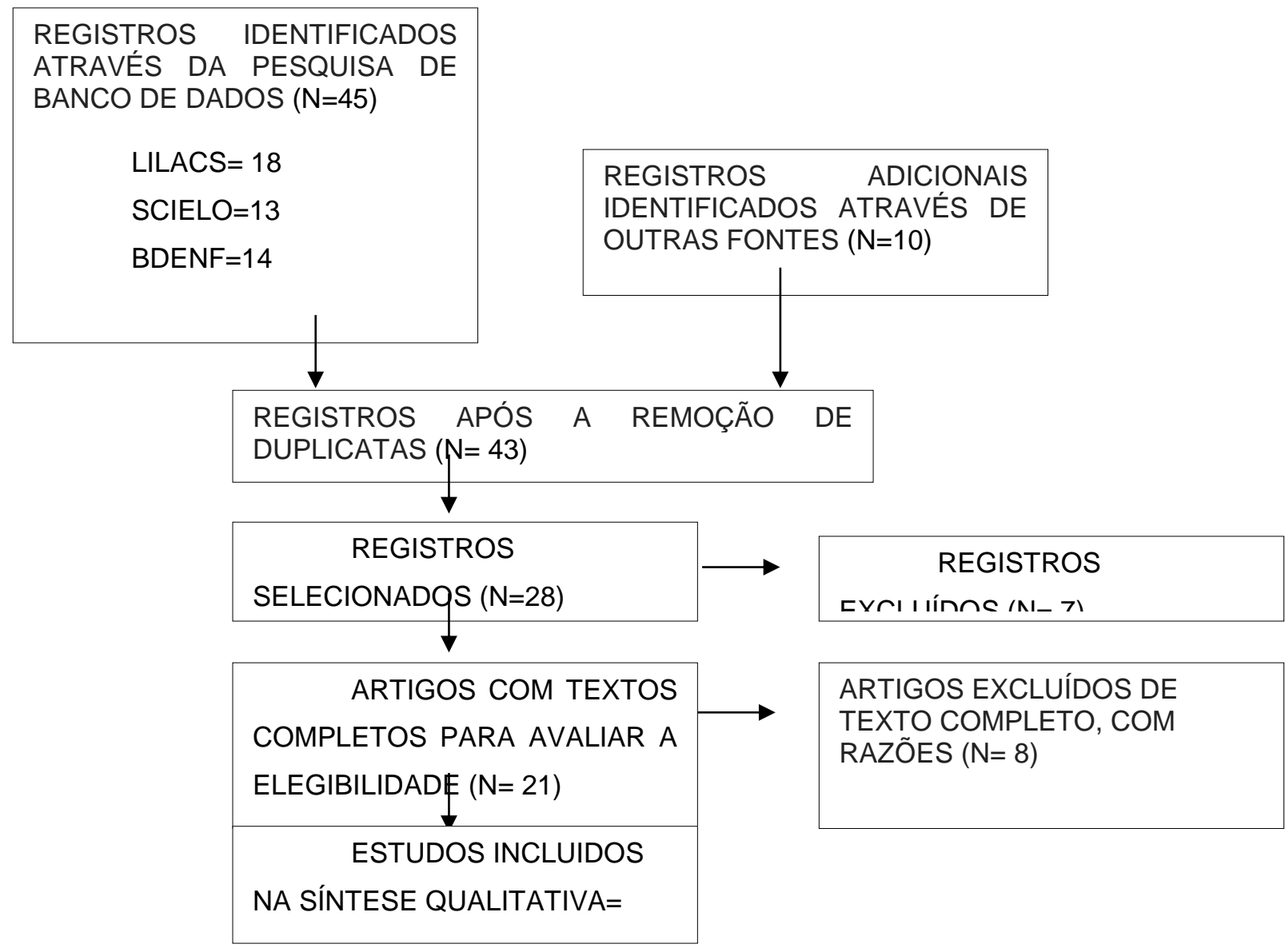

Os artigos selecionados foram agrupados e discutidos de acordo com a semelhança temática. 


\section{Resultados e Discussão}

A partir da análise dos dados coletados nos artigos selecionados, verificou-se que a área profissional que sobressaiu todas às demais com relação aos descritores, foi a de Enfermagem, representando $77,7 \%$ da amostra e com isso, demonstrando seu interesse em pesquisas referentes a saúde dos cuidadores de idosos. Outra área com mais publicações foi a de Geriatria e Gerontologia, representadas por 11,1\% cada, e por último, a área de Clínica Médica, também com $11,1 \%$. Quanto ao objetivo dos trabalhos, $77,7 \%$ da amostra são voltados para a saúde do cuidador e destes, 71,4\% correlaciona a sobrecarga dos cuidadores à saúde. Os 22,3\% restantes, abrangem outros assuntos como as relações intrafamiliares com o cuidador e a família do idoso, qualidade de vida relacionada ao cuidador e o processo de tornar-se cuidador.

Para melhor descrever os achados, optou-se pela demonstração informacional em quadro. $\mathrm{O}$ quadro 1 demonstra as seguintes informações: ordem de classificação do estudo, título, autores, ano de publicação, área da revista, delineamento da pesquisa e nível de evidência.

Quadro 1: Descrição dos achados segundo ordem de classificação do artigo, título, autores, ano, objetivo, área da revista, delineamento da pesquisa e nível de evidência científica.

\begin{tabular}{|c|c|c|c|c|c|c|c|}
\hline $\begin{array}{l}\text { Ordem de } \\
\text { classifi- } \\
\text { cação }\end{array}$ & Título & Autores & Ano & Objetivo & $\begin{array}{l}\text { Área da } \\
\text { pesquisa }\end{array}$ & $\begin{array}{c}\text { Delineamento da } \\
\text { pesquisa }\end{array}$ & $\begin{array}{l}\text { Nível de } \\
\text { evidencia }\end{array}$ \\
\hline A1 & $\begin{array}{l}\text { "Cuidar de um } \\
\text { familiar idoso } \\
\text { dependente no } \\
\text { domicílio: } \\
\text { reflexões para } \\
\text { os profissionais } \\
\text { da saúde" }\end{array}$ & $\begin{array}{l}\text { VIEIRA L; } \\
\text { NOBRE J. } \\
\text { R.S; } \\
\text { BASTOS C. } \\
\text { B. C; } \\
\text { TAVARES } \\
\text { K. O. }\end{array}$ & 2012 & $\begin{array}{l}\text { Apresentar } \\
\text { resultados } \\
\text { relacionados à } \\
\text { sobrecarga física e } \\
\text { mental relatadas } \\
\text { por cuidadores } \\
\text { familiares de } \\
\text { idosos } \\
\text { dependentes }\end{array}$ & $\begin{array}{l}\text { Geriatria e } \\
\text { gerontologia }\end{array}$ & $\begin{array}{l}\text { Estudo descritivo- } \\
\text { exploratório }\end{array}$ & Nível 2 \\
\hline A2 & $\begin{array}{l}\text { "Qualidade de } \\
\text { vida e saúde de } \\
\text { cuidadores de } \\
\text { idosos } \\
\text { longevos: } \\
\text { Interferências } \\
\text { intrafamiliares" }\end{array}$ & $\begin{array}{l}\text { NOVAIS N. } \\
\text { N; } \\
\text { SILVA L. W. } \\
\text { S; } \\
\text { GOLÇALVE } \\
\text { S L. H. T; } \\
\text { MENEZES T. } \\
\text { M. O. }\end{array}$ & 2013 & $\begin{array}{l}\text { Conhecer as } \\
\text { relações } \\
\text { intrafamiliares e } \\
\text { compreender } \\
\text { como interferem } \\
\text { na qualidade de } \\
\text { vida e saúde dos } \\
\text { familiares }\end{array}$ & Enfermagem & $\begin{array}{l}\text { Estudo Quanti- } \\
\text { qualitativo }\end{array}$ & Nível 4 \\
\hline A3 & $\begin{array}{l}\text { "Sobrecarga } \\
\text { em } \\
\text { cuidadores } \\
\text { familiares de } \\
\text { idosos: } \\
\text { associação } \\
\text { com } \\
\text { característica } \\
\text { s do idoso e } \\
\text { demanda de } \\
\text { cuidado" }\end{array}$ & $\begin{array}{l}\text { LOUREIRO } \\
\text { L. S. N; } \\
\text { FERNANDE } \\
\text { S G. M. F; } \\
\text { NÓBREGA } \\
\text { M. M. L. } \\
\text { RODRIGUE } \\
\text { Z R. A. P. }\end{array}$ & 2014 & $\begin{array}{l}\text { Estimar a } \\
\text { prevalência de } \\
\text { sobrecarga entre } \\
\text { cuidadores } \\
\text { familiares de } \\
\text { idosos } \\
\text { dependentes do } \\
\text { município de João } \\
\text { Pessoa, Paraíba, e } \\
\text { identificar sua } \\
\text { relação com } \\
\end{array}$ & Enfermagem & Transversal & Nível 6 \\
\hline
\end{tabular}




\begin{tabular}{|c|c|c|c|c|c|c|c|}
\hline & & & & $\begin{array}{l}\text { condições de } \\
\text { saúde, } \\
\text { funcionalidade e } \\
\text { demanda de } \\
\text { cuidado do idoso. }\end{array}$ & & & \\
\hline A4 & $\begin{array}{ll} & \text { "Idoso } \\
\text { situação de } & \text { de } \\
\text { dependência: } & \\
\text { estresse } & \\
\text { e coping do } & \\
\text { cuidador } & \\
\text { informal" } & \end{array}$ & $\begin{array}{l}\text { ROCHA B. } \\
\text { M. P; } \\
\text { PACHECO J. } \\
\text { E. P. }\end{array}$ & 2013 & $\begin{array}{l}\text { Estudar a relação } \\
\text { entre o estresse } \\
\text { psicológico } \\
\text { (estresse) e a } \\
\text { adaptação } \\
\text { psicológica } \\
\text { (coping) do } \\
\text { cuidador informal } \\
\text { do idoso em } \\
\text { situação de } \\
\text { dependência. }\end{array}$ & Enfermagem & Transversal & Nível 6 \\
\hline A5 & $\begin{array}{l}\text { "Perspectivas } \\
\text { atuais sobre a } \\
\text { sobrecarga do } \\
\text { cuidador em } \\
\text { saúde mental" }\end{array}$ & $\begin{array}{l}\text { CARDOSO } \\
\text { L; } \\
\text { VIEIRA M. } \\
\text { V. V; } \\
\text { RICCI M. A. } \\
\text { M; } \\
\text { MAZZA R. S. }\end{array}$ & 2012 & $\begin{array}{l}\text { Estudar a } \\
\text { sobrecarga entre } \\
\text { os cuidadores } \\
\text { informais e a } \\
\text { validação de } \\
\text { escalas } \\
\text { psicométricas, } \\
\text { destacando-se a } \\
\text { Escala de Zarit. }\end{array}$ & Enfermagem & $\begin{array}{l}\text { Revisão sistemática } \\
\text { da literatura }\end{array}$ & Nível 4 \\
\hline A6 & $\begin{array}{l}\text { "Estudos } \\
\text { nacionais sobre } \\
\text { cuidadores } \\
\text { familiares de } \\
\text { idosos: revisão } \\
\text { integrativa" }\end{array}$ & $\begin{array}{l}\text { OLIVEIRA } \\
\text { D. C; } \\
\text { D’ELBOUXI } \\
\text { I } \\
\text { D. M. J. }\end{array}$ & 2012 & $\begin{array}{l}\text { Realizar uma } \\
\text { revisão da } \\
\text { literatura } \\
\text { científica } \\
\text { brasileira sobre o } \\
\text { cui- } \\
\text { dador familiar do } \\
\text { idoso, com vistas } \\
\text { a buscar o } \\
\text { delineamento } \\
\text { dos trabalhos } \\
\text { publicados e os } \\
\text { temas abordados, } \\
\text { em âmbito } \\
\text { nacional e } \\
\text { internacional } \\
\end{array}$ & Enfermagem & $\begin{array}{l}\text { Revisão sistemática } \\
\text { da literatura }\end{array}$ & Nível 4 \\
\hline A7 & $\begin{array}{l}\text { "Qualidade de } \\
\text { vida } \\
\text { sobrecarga de } \\
\text { cuidadores } \\
\text { familiares de } \\
\text { idosos } \\
\text { dependentes" }\end{array}$ & $\begin{array}{l}\text { RODRIGUE } \\
\text { S J. E. G; } \\
\text { MACHADO } \\
\text { A. L. G; } \\
\text { VIEIRA N. } \\
\text { F. C; } \\
\text { FERNANDE } \\
\text { S A. F. C. F; } \\
\text { REBOLÇAS } \\
\text { C. B. A. }\end{array}$ & 2014 & $\begin{array}{l}\text { Avaliar a } \\
\text { qualidade de vida } \\
\text { relacionada à } \\
\text { saúde de } \\
\text { cuidadores } \\
\text { familiares de } \\
\text { idosos e relacioná- } \\
\text { la a sobrecarga de } \\
\text { trabalho de } 50 \\
\text { cuidadores de } \\
\text { idosos atendidos } \\
\text { em duas unidades } \\
\text { de Saúde da } \\
\text { Família }\end{array}$ & Enfermagem & Estudo qualitativo & Nível 4 \\
\hline A8 & $\begin{array}{l}\text { "O impacto da } \\
\text { doença crônica } \\
\text { no cuidador" }\end{array}$ & $\begin{array}{l}\text { FERREIRA } \\
\text { H. P. F; } \\
\text { MARTINS L. } \\
\text { C; } \\
\text { BRAGA A. } \\
\text { L; } \\
\text { GARCIA M. } \\
\text { L. B. }\end{array}$ & 2012 & $\begin{array}{l}\text { Investigar o } \\
\text { impacto da doença } \\
\text { crônica na vida do } \\
\text { familiar cuidador } \\
\text { do paciente } \\
\text { crônico. }\end{array}$ & $\begin{array}{l}\text { Clínica } \\
\text { médica }\end{array}$ & Transversal & Nível 6 \\
\hline A9 & $\begin{array}{l}\text { "Investigação } \\
\text { do impacto da } \\
\text { doença crônica } \\
\text { na vida do } \\
\text { familiar } \\
\text { cuidador do }\end{array}$ & $\begin{array}{l}\text { COSTA R. S. } \\
\text { C; } \\
\text { BRITO T. R. } \\
\text { P; } \\
\text { VIANA A. S. } \\
\text { V; }\end{array}$ & 2013 & $\begin{array}{l}\text { Descrever a } \\
\text { estrutura e função } \\
\text { das redes de apoio } \\
\text { social de idosos } \\
\text { com alterações } \\
\text { cognitivas que } \\
\text { residem em }\end{array}$ & Enfermagem & $\begin{array}{l}\text { Transversal, } \\
\text { descritivo baseado } \\
\text { no método } \\
\text { quantitativo }\end{array}$ & Nível 6 \\
\hline
\end{tabular}




\begin{tabular}{|c|c|c|c|c|c|c|c|}
\hline & $\begin{array}{l}\text { paciente } \\
\text { crônico." }\end{array}$ & $\begin{array}{l}\text { PAVARINI } \\
\text { S. C. L. }\end{array}$ & & $\begin{array}{l}\text { contexto de alta } \\
\text { vulnerabilidade } \\
\text { social, e de seus } \\
\text { cuidadores } \\
\text { familiares }\end{array}$ & & & \\
\hline A10 & $\begin{array}{l}\text { "Cuidado } \\
\text { terapêutico de } \\
\text { enfermagem: } \\
\text { transições da } \\
\text { sexualidade do } \\
\text { cônjuge-cuidador } \\
\text { do idoso" }\end{array}$ & $\begin{array}{l}\text { LIMA C. F. } \\
\text { M; } \\
\text { CALDAS P. } \\
\text { C; } \\
\text { SANTOS I; } \\
\text { TROTTE L. } \\
\text { A. C; } \\
\text { SILVA B. M. } \\
\text { C. }\end{array}$ & 2017 & $\begin{array}{l}\text { Compreender as } \\
\text { transiç̃oes } \\
\text { vivenciadas, suas } \\
\text { condições e os } \\
\text { padrões de } \\
\text { resposta esperados } \\
\text { a mudanças na } \\
\text { sexualidade do } \\
\text { cônjuge-cuidador } \\
\text { do idoso em } \\
\text { processo } \\
\text { demencial. }\end{array}$ & Enfermagem & $\begin{array}{l}\text { Exploratório de } \\
\text { abordagem } \\
\text { qualitativa }\end{array}$ & Nível 6 \\
\hline A11 & $\begin{array}{l}\text { "Resignificação } \\
\text { da vida do } \\
\text { cuidador do } \\
\text { paciente idoso } \\
\text { com câncer" }\end{array}$ & $\begin{array}{l}\text { ANJOS A. C. } \\
\text { Y; } \\
\text { ZAGO M. M. } \\
\text { F. }\end{array}$ & 2014 & $\begin{array}{l}\text { Analisar o } \\
\text { processo de } \\
\text { tornar-se cuidador } \\
\text { de idoso com } \\
\text { câncer, submetido } \\
\text { à quimioterapia, } \\
\text { no contexto } \\
\text { domiciliar. }\end{array}$ & Enfermagem & $\begin{array}{l}\text { Entrevistas semi- } \\
\text { estruturadas de } \\
\text { abordagem } \\
\text { qualitativa }\end{array}$ & Nível 4 \\
\hline A12 & $\begin{array}{l}\text { "Os Desafios do } \\
\text { Cuidar: Revisão } \\
\text { Bibliográfica, } \\
\text { Sobrecargas e } \\
\text { Satisfaç̃̃es do } \\
\text { Cuidador de } \\
\text { Idosos" }\end{array}$ & $\begin{array}{l}\text { FALEIROS } \\
\text { A. H; } \\
\text { SANTOS C. } \\
\text { A; } \\
\text { MARTINS C. } \\
\text { R; } \\
\text { HOLANDA } \\
\text { R. A. H; }\end{array}$ & 2015 & $\begin{array}{l}\text { Identificar as } \\
\text { sobrecargas e } \\
\text { satisfações de } \\
\text { cuidadores de } \\
\text { idosos e destacar } \\
\text { o contexto dos } \\
\text { eventos } \\
\text { estressores e dos } \\
\text { mediadores } \\
\text { sociais e pessoais. }\end{array}$ & Enfermagem & Estudo qualitativo & Nível 4 \\
\hline A13 & $\begin{array}{l}\text { "O Perfil } \\
\text { Socioepidemiol } \\
\text { ógico e a } \\
\text { Autopercepção } \\
\text { dos Cuidadores } \\
\text { Familiares } \\
\text { sobre a Relação } \\
\text { Interpessoal e o } \\
\text { Cuidado com } \\
\text { Idosos" }\end{array}$ & $\begin{array}{l}\text { SANTOS B. } \\
\text { E; } \\
\text { KOETZ L. C. } \\
\text { E. }\end{array}$ & 2017 & $\begin{array}{l}\text { Identificar o perfil } \\
\text { dos cuidadores } \\
\text { informais de } \\
\text { idosos } \\
\text { dependentes } \\
\text { residentes em } \\
\text { domicílio, } \\
\text { assistidos pelo } \\
\text { programa } \\
\text { Estratégias e } \\
\text { Saúde da Família } \\
\text { (ESF) } \\
\end{array}$ & Fisioterapia & $\begin{array}{l}\text { Estudo descritivo de } \\
\text { abordagem } \\
\text { qualitativa }\end{array}$ & Nível 4 \\
\hline
\end{tabular}




\section{A saúde mental dos cuidadores}

A partir na análise criteriosa dos trabalhos, foi possível perceber os fatores que interferem na saúde dos cuidadores de idosos, para os autores do estudo 1 (A1), a sobrecarga e a responsabilidade que um idoso dependente demanda, influencia, de forma negativa nas relações familiares, bem como o desenvolvimento de doenças físicas e mentais.

O estudo 6 (A6) constatou que os a consequências da sobrecarga de trabalho dos cuidadores pode desenvolver nestes indivíduos, distúrbios psicoemocionais decorrentes do grande tempo de dedicação ao cuidado, falta de informação do cuidador, a presença de morbidades como depressão e o grau de dependência em que o idoso se encontra.

$\mathrm{Na}$ maioria dos artigos pesquisados (85\%), em foi abordada a questão da sobrecarga de trabalho do cuidador, deste total, $80 \%$ apresentaram que a maior carga de estresse é em cuidadores informais, que prestam cuidados primários, cuidadores familiares e cuidadores idosos.

No que diz respeito a qualidade de vida do cuidador, os estudos A2 e A7 trazem que, em média, $90 \%$ dos cuidadores são do sexo feminino, destes, $52 \%$ são filhas destes idosos, dentre estes, $42 \%$ revelam que sua saúde está pior desde que tornaram-se cuidadores familiares, os aspectos que eles demonstram estarem mais afetados são as relações sociais, os aspectos emocionais, dores e a saúde mental.

Para o estudo 12 (A12), cuidar de um idoso dependente, além de trazer a sobrecarga biopsicossocial e emocional para o cuidador, com o passar do tempo e a exposição prolongada a essas condições, torna-se fator agravante ao surgimento e agravamento de doenças relacionadas.

61,53\% dos artigos destacam a importância da promoção de orientações pela equipe de saúde na vida do cuidador, para melhor auxiliá-lo na prestação dos cuidados sem o sobrecarregar. Assim como também, prestar atendimento para ouvir o que essas pessoas tem a dizer, dando a oportunidade para que desabafem sobre quais suas maiores dificuldades e sintomas, podendo assim, intervir com prescrições de enfermagem para a melhora na qualidade de vida destes, capacitando o cuidador para a realização das atividades que o idoso não têm condições de realizar e, delegar a ele o que ainda é capaz, estimulando assim sua independência e reduzindo a sobrecarga do cuidador. 


\section{Considerações Finais}

Por meio deste estudo, constatou-se a escassez de pesquisas nacionais sobre o déficit de autocuidado de cuidadores de idosos, bem como de trabalhos inovadores para a área. São poucas as pesquisas que tem base exploratória e resolutiva do problema, na grande maioria há apenas a discussão sobre o quanto a qualidade de vida destes cuidadores é comprometida devido à sobrecarga de responsabilidades e afazeres para com o idoso, deixado a si mesmo em outro plano e não cuidando de sua própria saúde.

Nota-se também que há um déficit em programas de saúde voltados para esse público e com isso, a necessidade de se avançar em políticas e capacitações com práticas que trabalhem na melhoria da saúde dessas pessoas, diminuindo o estresse e a sobrecarga.

Neste sentido, pensando-se na saúde pública e nos profissionais de saúde que acompanham as famílias, reforça-se que cabe a cada um desses responsáveis pela comunidade abrangida por sua Unidade Básica de Saúde (UBS), estabelecer vínculos terapêuticos com essa família, favorecendo melhorias na qualidade de vida, tanto do cuidador, quanto do idoso, trabalhar junto com toda a equipe de saúde do local para encaminhar essas pessoas para os profissionais adequados, principalmente o cuidador, para que este possa cuidar de sua saúde e assim, estar disposto e com as saúdes física e mental íntegras, para maior eficácia do seu cuidado para com o idoso.

\section{Referências}

ANJOS, A.C.Y.; ZAGO, M.M.F. Ressignificação da vida do cuidador do paciente idoso com câncer. Rev Bras Enferm. v. 67, n. 5, p. 752-8, 2014.

BRASIL. Ministério da Saúde; Secretaria de Atenção à Saúde. Departamento de Ações Programáticas e Estratégicas. Caderneta de saúde da pessoa idosa. Brasília, v. 3, 2014.

CARDOZO, L. et al. Perspectivas atuais sobre a sobrecarga do cuidador em saúde mental. Rev Esc Enferm, São Paulo, 2012.

COSTA, R.S. et al. Apoio social em contexto de pobreza: Estudando idosos com alterações cognitivas e seus cuidadores familiares. Rev. cuid. fundam. online, 2013. 
FALEIROS, A.H. et al. Os Desafios do Cuidar: Revisão Bibliográfica, Sobrecargas e Satisfações do Cuidador de Idosos. Janus, Lorena, n. 21, 2015.

FERREIRA, H.P. et al. O impacto da doença crônica no cuidador. Rev Bras Clin Med. São Paulo, 2012.

GALVÃO, T.F.; PENSANI, T.S.A.; HARRAD, D. Principais itens para relatar Revisões sistemáticas e Meta-análises: A recomendação PRISMA. Epidemiol. Serv. Saúde. Brasília, v. 24, n. 2, 2015.

LIMA, C.F.M. et al. Therapeutic nursing care: transition in sexuality of the elderly caregiving spouse. Rev Bras Enferm. v. 70, n. 4, p. 673-81, 2017.

LOUREIRO, L.S.N. et al. Sobrecarga em cuidadores familiares de idosos: associação com características do idoso e demanda de cuidado. Rev. bras. enferm. Brasília, v. 67, n. 2, 2014.

NOVAIS, N.N. et al. Qualidade de vida e saúde de cuidadores de idosos longevos: interferências intrafamiliares. Revista Baiana de Enfermagem. v. 27, n. 1, 2013.

OLIVEIRA, D.C.; D'ELBOUX, M.J. Estudos nacionais sobre cuidadores familiares de idosos: revisão integrativa. Rev Bras Enferm, Brasília, 2012.

ROCHA, B.M.P.; PACHECO, J.E.P. Idoso em situação de dependência: estresse e coping do cuidador informal. Acta paul. enferm. São Paulo, v. 26, n. 1, 2013.

RODRIGUES, J.E.G. et al. Qualidade de vida e sobrecarga de cuidadores familiares de idosos dependentes. Cienc. enferm. v .20, n. 3, 2014.

SANTOS, B.E.; KOETZ, L.C.E. O Perfil Socioepidemiológico e a Autopercepção dos Cuidadores Familiares sobre a Relação Interpessoal e o Cuidado com Idosos. Rev ACRED. v. 7, n. 13, 2017.

SOUZA, M.; SILVA, M.D.; CARVALHO, R. Revisão integrativa: o que é e como fazer. Einstein. São Paulo, v. 8, n. 1, 2010.

VIEIRA, L. et al. Cuidar de um familiar idoso dependente no domicílio: reflexões para os profissionais da saúde. Rev. bras. geriatr. gerontol. Rio de Janeiro, v. 15, n. 2, 2012.

\section{Como citar este artigo (Formato ABNT):}

RAMOS, Raquel Gouveia; LEITE-SALGUEIRO, Claudia Daniele Barros; PEREIRA, Juliane da Silva; BARBOSA, Luciclaudio da Silva; LOBATO, Luzineide. Cuidadores de Idosos e o Déficit no Autocuidado. Id on Line Rev.Mult. Psic., 2018, vol.12, n.41, p.1083-1085. ISSN: 19811179.

Recebido: 11/07/2018.

Aceito: $26 / 07 / 2018$ 